\title{
Diabetes: Avaliação do hábito alimentar de escolares
}

\author{
Diabetes: Evaluation of food habit of school \\ Jordana Silva Pereira.
}

Como citar esse artigo. Pereira JS. Diabetes: Avaliação do hábito alimentar de escolares. Revista Saúde. 2013 Jan./Dez.; $04 \quad(1 / 2)$ : 23-28.

\begin{abstract}
Resumo
O Diabetes é uma doença cujos índices têm aumentado nos últimos anos. O Diabetes tem sido tratado como um problema de saúde pública, pois acarreta consequências sociais, psicológicas e de saúde que afetam crianças, adolescentes, adultos e idosos e aumenta a cada ano. Objetivos: é analisar os lanches que os alunos levam, comparando os lanches de duas escolas, uma pública e uma privada, a Escola Municipal Condessa do Rio Novo e Colégio Cenecista Sul Paraibano. Materiais e Métodos: foi desenvolvido um questionário algumas perguntas relacionadas à alimentação escolar, foram entrevistados 40 alunos, sendo 20 de cada escola. . Resultados: Os resultados mostram que os alunos, principalmente da escola privada, têm maus hábitos alimentares, levam uma vida sedentária, muitos alunos estão abaixo do peso ideal e alguns estão obesos e diabéticos, resultados de uma alimentação rica em gorduras, sal e alimentos industrializados. Conclusão: este trabalho mostra a importância da alimentação dos alunos, e as consequências da má alimentação, que pode causar diabetes.

Palavras-chave: Diabetes. Alimentação. Hábitos saudáveis.
\end{abstract}

\begin{abstract}
Diabetes is a disease whose rates have increased in recent years. Diabetes has been treated as a public health problem because it has social, psychological and health consequences that affect children, adolescents, adults and the elderly and increases every year. Objectives: is to analyze what students bring snacks, snacks comparing the two schools, one public and one private, the City College of New River Countess and Cenecista College South Paraiba. Materials and Methods: A questionnaire was developed some questions related to school feeding, 40 students were interviewed, 20 from each school. . Results: The results show that students, especially the private school, have poor eating habits, lead a sedentary life, many students are underweight and some are obese and diabetic, results of a diet rich in fats, salt and processed foods. Conclusion: This study shows the importance of feeding the students, and the consequences of poor diet, which can cause diabetes.

Keywords: Diabetes. Diet. Healthy habits.
\end{abstract}

\section{Introdução}

O Diabetes Mellitus tem sido um problema individual e de saúde pública que atinge muitas pessoas de todas as classes sociais, que já alcança proporções epidêmicas, pois tem aumentado exageradamente nos últimos anos. (Rodrigues et al., 2006).

Este aumento está diretamente relacionado à maior urbanização, aumento da obesidade e sedentarismo, ao crescimento e envelhecimento populacional, e aos maus hábitos alimentares (Wild et al., 2004).

O índice de pessoas diabéticas é maior em países desenvolvidos, e estima que continuará sendo. Pesquisas mostram que até 2030 ocorrerá um aumento proporcional em países em desenvolvimento, que concentrara $2 / 3$ dos indivíduos diabéticos. A estimativa é de $70 \%$ de aumento na prevalência em países em desenvolvimento, contra $42 \%$ nos países desenvolvidos (King; Aubert; Herman, 1998; Wild et al., 2004).

Entre os países com maior número de diabéticos, a Índia e a China, países populosos que não são desenvolvidos, estão em primeiro lugar, logo após o
Estados Unidos. O Brasil aparece em oitavo lugar, segundo as estimativas dos anos de 1995 para 2030, indo para o sexto lugar até $2030 \mathrm{com}$ aproximadamente 11,3 milhões de doentes. (Wild et al., 2004).

Em 2005 havia 8 milhões de diabéticos no Brasil, sendo $4,5 \%$ do total da população (SBD, 2009). O Diabetes Mellitus tornou-se hoje uma epidemia mundial, tornando um grande problema de saúde pública (Brasil, 2006).

Diabetes Mellitus I (DMI) configura-se pela hiperglicemia crônica, causada por muitos fatores, ambiental e genética, que caracteriza pela falta de insulina, ocasionando alterações no metabolismo de gorduras, carboidratos e proteínas (SBD, 2006).

O Diabetes Tipo I (DMI) é caracterizado pelo aumento do açúcar no sangue devido à ausência de insulina, uma substância que queima o açúcar. Na maioria das vezes, a pessoa descobre por acaso em um exame de sangue ou de urina, sem que o paciente apresente sintomas da doença. Há alguns sintomas característicos desta doença, poligamia (apetite exagerado), polidipsia (sede excessiva), poliúria (eliminação de grande 
quantidade de urina) e perda de peso (Pedroza, 2000).

O Diabetes Tipo II é uma doença crônica de etiologia multifatorial, de prevalência crescente. Tratase de um problema em progressão como manifestação da transição epidemiológica que, com ritmos diferentes, acomete praticamente todos os países do mundo (Lieberman, 2003).

\section{Materiais e Métodos}

Foi realizado estudo transversal através da coleta de dados, com alunos de duas escolas de Paraíba do Sul, sendo uma pública e outra privada.

A pesquisa foi realizada na Escola Municipal Condessa do Rio Novo no dia 03 de dezembro de 2012 e no Colégio Cenesista Sul Paraibano no dia 06 de dezembro de 2012, com alunos entre 8 e 13 anos, do Ensino Fundamental. O desenvolvimento foi feito com alunos nesta faixa etária devido à grande maioria levar lanches para consumir durante o recreio. $\mathrm{O}$ total de entrevistados foram 40 alunos, sendo 20 de cada escola.

Inicialmente foi realizado o contato com a direção da escola, tendo este autorizado a realização da pesquisa. A coleta de dados, só foi realizada após a autorização da escola.

Apesquisa foi realizada através de um questionário com questões, o mesmo foi passado na Escola pública Condessa do Rio novo, e na privada Colégio Cenecista Sul Paraibano e teve como objetivo identificar e comparar os lanches consumidos nas duas escolas.

A identidade dos alunos foi preservada, e suas respostas compiladas em gráficos.

\section{Resultados}

A figura 1 mostra o percentual de alunos que trazem lanches de casa da escola Condessa do Rio Novo, $4 \%$ dos alunos responderam que levam lanches de casa, $26 \%$ não levam e $70 \%$ levam as vezes. Observando os dados acima, revela que $50 \%$ dos alunos do Colégio Cenecista Sul Paraibano levam lanches de casa, 15\% não levam lanches e 35\% levam lanches as vezes.

A figura 2 mostra uma comparação entre o percentual de lanches que os alunos preferem trazer

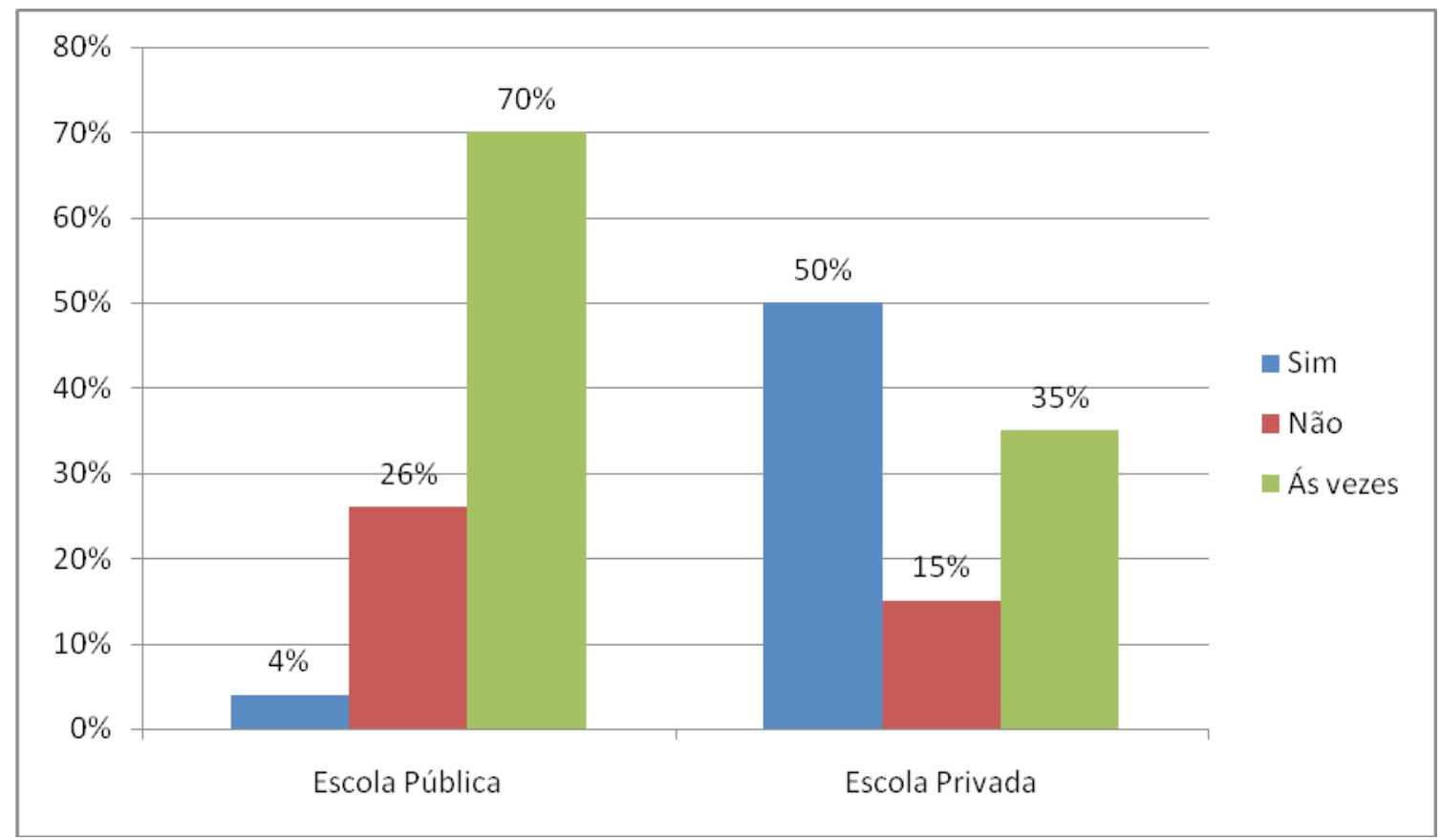

Figura 1. Percentual de Alunos que trazem lanches

para se alimentar no horário do recreio, entre salgado, fruta, bala, bolo, sanduíches e biscoito.
Através da figura 3 podemos analisar a preferência dos alunos de cada escola em relação a suco, 


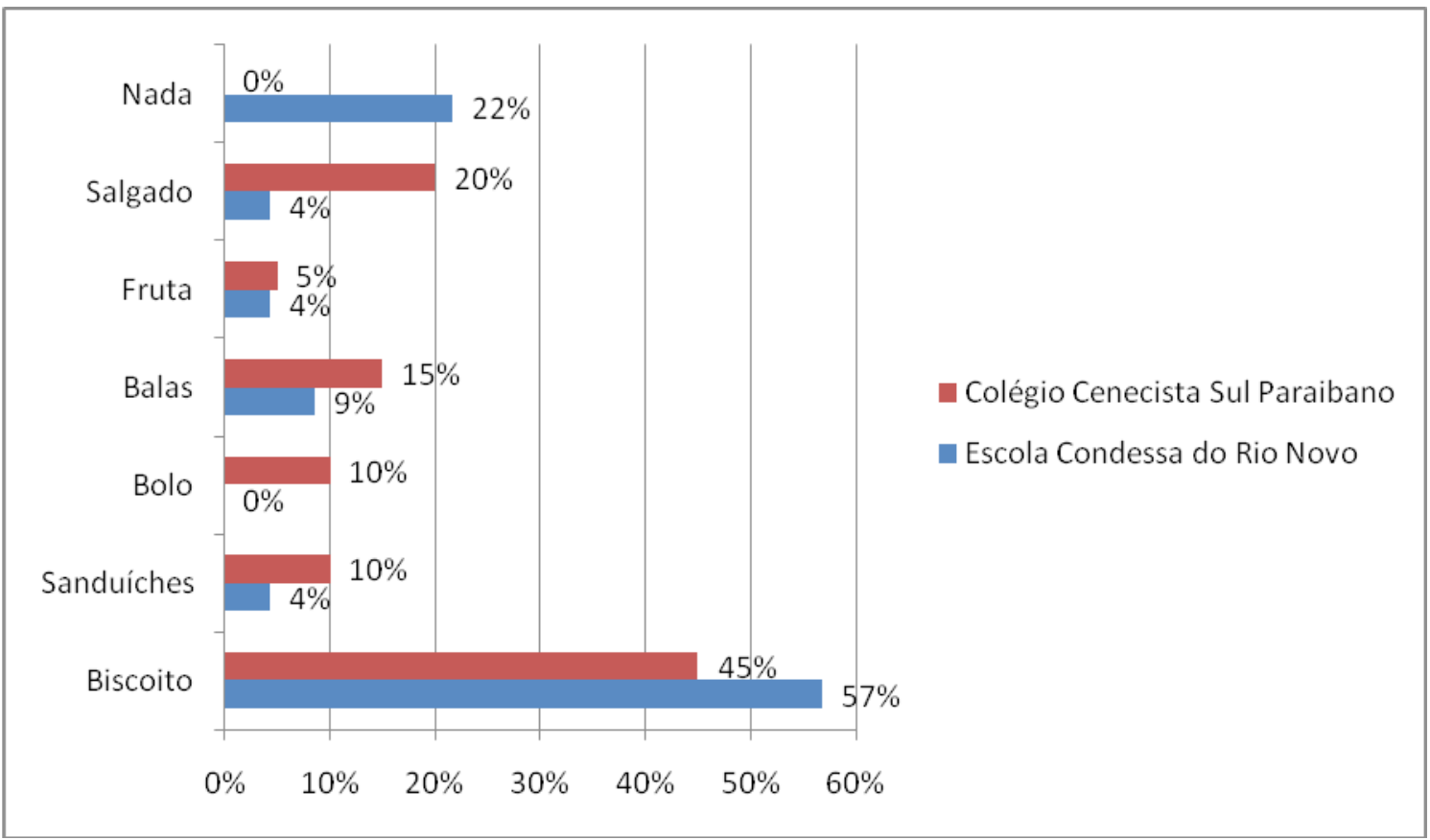

Figura 2. Percentual de Lanches que os Alunos trazem de Casa em ambas as escolas

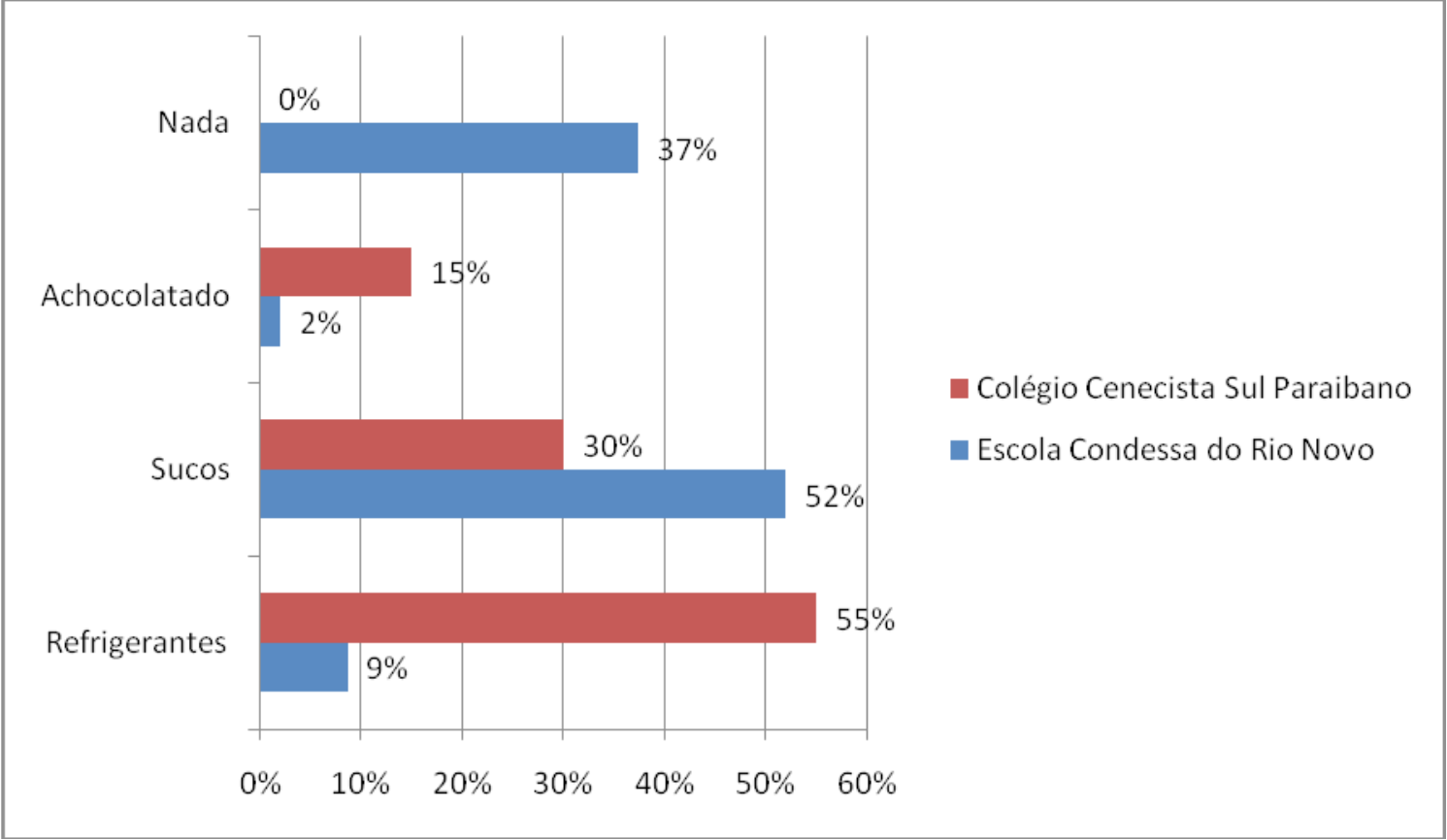

Figura 3. Percentual de bebidas que os Alunos trazem de Casa em ambas as escolas

achocolatado e refrigerantes.

Na escola pública $100 \%$ dos alunos não compram na cantina, por não haver cantina na escola.

Dados da figura 4 revelam os alimentos que os alunos, não gostam de comer,e que geralmente não fazem parte da sua alimentação na escola.

Analisando a figura 5, podemos ver o cálculo do Índice de Massa Corporal (IMC) dos alunos de ambas as escolas, e pode-se comparar o nível de peso de cada um deles. Na Escola Pública Condessa do Rio Novo 39\% dos alunos entrevistados estão abaixo do peso normal, $53 \%$ estão no peso ideal e $8 \%$ estão acima do peso. Já no Colégio Privado Sul Paraibano $45 \%$ estão abaixo do peso normal, $30 \%$ estão com o peso ideal e $25 \%$ estão acima do peso ideal.

\section{Discussão}

Neste trabalho foram mostrados os resultados obtido através de um questionário realizado com alunos da Escola Pública Condessa do Rio Novo e do Colégio 


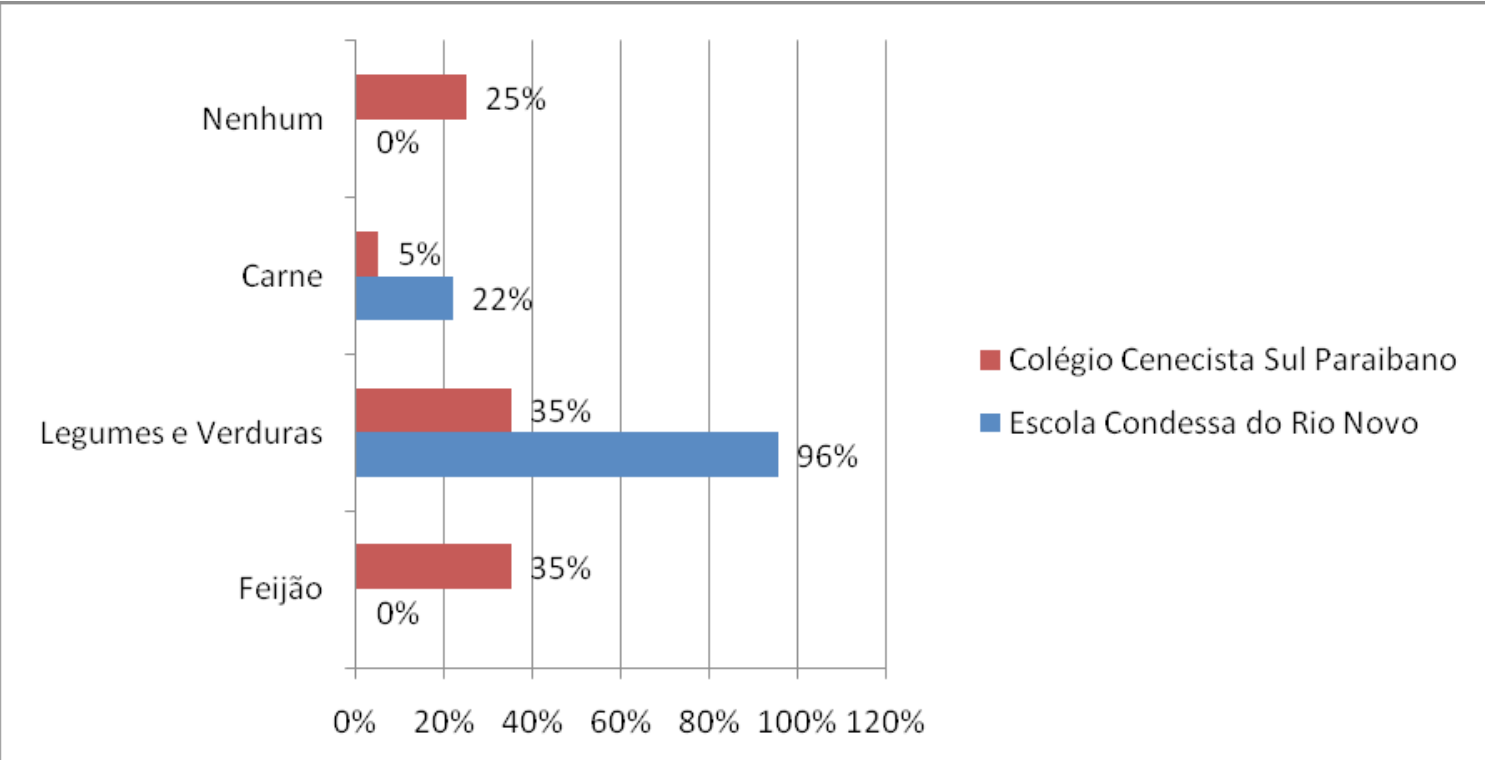

Figura 4. Percentual de alimentos que os Alunos não gostam de comer em ambas as escolas

Privado Sul Paraibano, ambas situado em Paraíba do Sul. O objetivo deste trabalho é analisar os lanches que os alunos levam. Os resultados mostram que os alunos principalmente da escola privada, tem maus hábitos alimentares, e levam uma vida sedentária, e muitos estão abaixo do peso ideal e podem vir a desenvolver o diabetes.

A avaliação da frequência de alunos que levam lanche para a escola (Figura 1) mostrou que na escola pública $70 \%$ dos alunos levam às vezes, $26 \%$ não levam

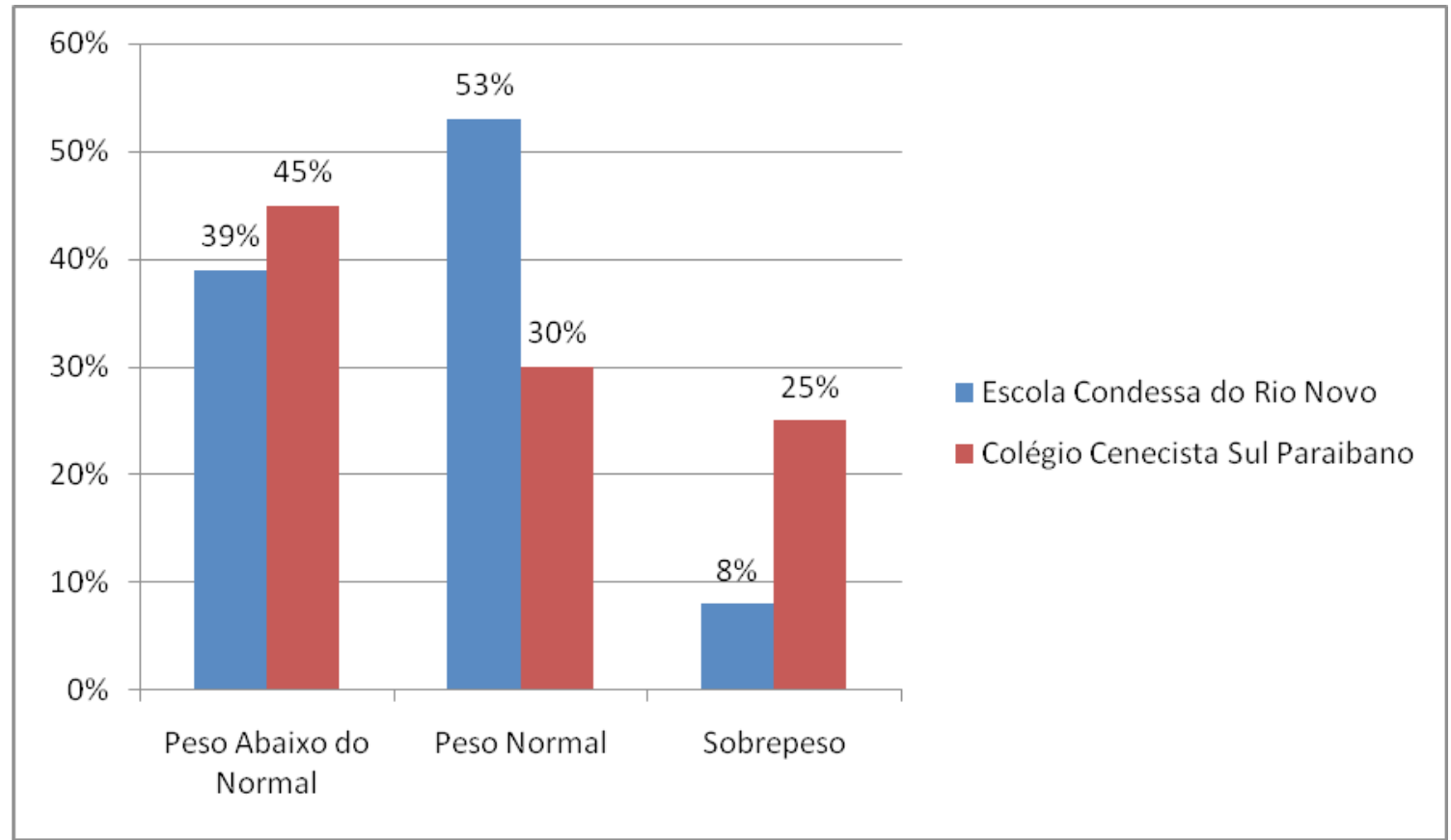

Figura 5. Cálculo do IMC - Índice de Massa Corporal em ambas as escolas

e $4 \%$ levam. Já na escola privada $35 \%$ levam às vezes, $15 \%$ não levam e $50 \%$ levam. É na infância que são constituídos os hábitos alimentares que as crianças irão levar para o resto da vida, por isso é importantes que a escola esteja presente auxiliando essa construção de hábitos saudáveis. A escola pública oferece a merenda, que é uma alimentação saudável, pois o cardápio é desenvolvido por nutricionista, sendo assim grande parte dos alunos se alimentam com a merenda oferecida pela escola, por isso não levam lanches de casa com grande frequência, já na escola privada não é oferecido merenda e os alunos levam lanches de casa. As necessidades nutricionais das crianças são determinadas pelo metabolismo basal, pelo ritmo de seu crescimento corporal, pelo nível de atividades físicas realizadas e pelo repouso, também pelo clima que vive e aspectos genéticos. Para se definir o valor energético do lanche das crianças na escola deve ser ter noção do tempo que esta irá passar na escola. A alimentação das crianças também sofre influências dos pais, já que são 
estes que compram e arrumam os lanches das crianças. O comportamento alimentar influencia muito para as crianças não desenvolverem a síndrome alimentar.

Segundo estudo realizado por Teixeira (2008), encontrou os seguintes resultados 26,5\% trazem lanches, 16,3 não trazem e $57,1 \%$ às vezes trazem. Entre os diversos alimentos que os alunos trazem, são suco, salgado frito, achocolatado e alimentos industrializados.

Hábitos alimentares incorretos contribuem para o aumento do peso, além de influenciar no crescimento e desenvolvimento e antecipa algumas doenças como hipertensão e diabetes tipo 2, aumentar o risco de aparecimento de doenças cardíacas e osteoporose (Falcão; Gomes; Schimitz, 2006)

A escola pública segue o Programa Nacional de Alimentação Escolar (PNAE), programa onde são oferecidos recursos em dinheiro para compra de alimentos saudáveis para as merendas das escolas públicas no Brasil, um programa antigo desenvolvido pelo Governo Federal. Desenvolvido em 1954 e criando forças em 1955, dando aos alunos de rede pública o direito à alimentação de acordo com o Art. 208 da Constituição Federal. Este programa busca melhores condições de alimentação para as crianças, além de melhores condições de aprendizagem e combate à fome (Brasil, 2008).

Quanto a preferência dos lanches que os alunos trazem de casa (Figura 3), em ambas as escolas os alunos preferem alimentos que não são saudáveis como biscoitos, balas, sanduíches, salgados, embora a frequência na escola pública seja bem menor em relação a escola privada. E a preferência por frutas é pequena $4 \%$ na escola pública e $5 \%$ na escola privada, e $22 \%$ dos alunos da escola pública preferem comer a merenda oferecida pela escola. O lanche alimentar saudável é um desafio na alimentação de crianças. Já as bebidas $2 \%$ dos alunos da escola pública preferem achocolatado, $37 \%$ preferem não levar nada, $52 \%$ preferem sucos e $9 \%$ preferem refrigerantes. Na escola privada $15 \%$ preferem achocolatado, $30 \%$ preferem sucos e $55 \%$ preferem refrigerantes e $0 \%$ não levam nada. Em relação as bebidas os alunos da escola pública possuem melhores hábitos que a escola particular, tendo em vista que preferem sucos.

Segundo estudo realizado por Fernandes (2006), as crianças têm preferência por alimentos, cujo valor calórico e nutricional ultrapassa as recomendações preconizadas na legislação.

Já Carvalho (2001), em sua pesquisa, constatou a presença marcante de alimentos gordurosos, ricos em açúcares, com pouca fibra e de pouco valor nutricional, e a existência de hábito alimentar inadequado pelos estudantes.

De acordo com Abrantes (2002), a alimentação inadequada pode levar a distúrbios nutricionais, como sobrepeso, obesidade e diabetes que estão níveis elevados na faixa etária pediátrica.

Observando dados da figura 4, revela que 35\% dos alunos da escola privada não gostam de legumes e verduras, e $96 \%$ da escola pública também não gostam um dado preocupante. Os dados mostram que alunos das escolas públicas têm maior preferência por alimentos saudáveis, enquanto os da escola privada preferem alimentos industrializados, massas e gorduras, deixando de lado frutas e verduras.

De acordo com a figura 5, foi calculado o Índice de Massa Corporal (IMC), na escola pública 39\% dos alunos estão abaixo do peso e na escola privada $45 \%$, na escola pública mais da metade dos alunos estão no peso ideal reflexo de uma alimentação mais saudável do que na escola privado, onde apenas $30 \%$ estão no peso ideal, e poucos estão acima do peso, não foi encontrado nenhum caso de obesidade durante o desenvolvimento deste trabalho, porém alunos que estão acima do peso devem seguir uma dieta para não se tornarem obesos. Os alunos da escola pública provavelmente estão mais saudáveis, por não ter acesso á cantina e aos alimentos hipercalóricos, pois se alimentam da merenda oferecida pela escola em cumprimento à lei $\mathrm{n}^{\mathrm{o}} 11.947$, de 16 de junho de 2009, que trata de diretrizes da alimentação escolar (Legislação Educacional - PCNs).

\section{Conclusão}

De acordo com os dados apresentado, concluise que, os alunos da escola pública possuem melhores hábitos alimentares. Maior proporção de alunos da escola privada apresenta IMC acima do desejável.

A maioria dos alunos se alimenta de produtos industrializados, doces, massas e gorduras, o que pode contribuir pra se tornarem diabéticos. Os alunos de escola particular estão mais propensos a desenvolver diabetes e obesidade, que os alunos de escola pública.

\section{Referências}

ABRANTES, M.M.; LAMOUNIER, J.A.; COLOSISMO, E. A Prevalência de sobrepeso e obesidade em crianças e adolescentes das regiões Sudeste e Nordeste. Jornal de Pediatria, Rio de Janeiro, v.78, n.4, p.335-340, 2002.

Brasil.Ministério da Saúde. Secretaria de Atenção À Saúde. Departamento de Atenção Básica. Diabetes Mellitus/Ministério da Saúde. Secretaria de Atenção à Saúde. Departamento de Atenção Básica - Brasília: Ministério da Saúde, 2006 56p.

BRASIL, Ministério do Desenvolvimento Social. Disponível em: http:/ www.mds.gov.br/secretarias/secretaria01_33. asp. Acesso em: 11 de dezembro de 2013

CARVALHO, Cecilia Maria Resende Gonçalves de et al., Consumo alimentar de adolescentes matriculados em um colégio particular de Teresina, Piauí, Brasil. Rev. Nutr. [online]. 2001, vol.14, n.2, pp. 85-93.

FALCAO-GOMES, Renata Couto; COELHO, Andréa Araciaba Soares; SCHMITZ, Bethsáida de Abreu Soares. Caracterização dos estudos de avaliação do consumo alimentar de pré-escolares. Rev. Nutr. Campinas, v. 19 , n. $6,200$. 
FERNANDES, F.M. Alimentação e nutrição entre escolares: caso dos alunos de uma escola do município de Vitória-ES. 2006. 49p. Monografia (Especialização em Nutrição Clínica) - Universidade Veiga de Almeida, Vitória, 2006

KING, H., AUBERT, R.E., HERMAN, W.H., 1998. Global burden of diabetes.Diabetes Care, 21 (21): 1414-1431.

LIEBERMAN, L.S. Dietary, evolutionary, and modernizing influences on the prevalence of type 2 diabetes. Ann Rev Nutr, v. 23, p.: 345-77, 2003.

PEDROZA, F. G. Diabetes para diabéticos, 2000. Disponível em http:// www.nead.unama.br/site/bibdigital/monografias/diabetes defesa.pdf Acesso em 07/09/2012 ás 13:14 horas.

RODRIGUES, T. C. et al., O controle do Diabetes mellitus em usuários de unidade básica de saúde, Campinas, SP, Ciênc. Cuid. Saúde. Maringá. v. 5, n. 1, p. 41-49, Jan.2006.

SBD. SOCIEDADE BRASILEIRA DE DIABETES. Dados sobre Diabetes Mellitus no Brasil. 2006.

TEIXEIRA, Patricia. O PROGRAMA NACIONAL DE ALIMENTAÇÃO ESCOLAR NO MUNICÍPIO DE CRICIÚMA, SC: PERCEPÇÃO DOS ESCOLARES. Trabalho de Conclusão de Curso. Universidade do Extremo Sul Catarinense, Criciúma, 2008.

WILD, S., ROGLIC, G., GREEN, A., SIRCREEN, R., KING, H., 2004. Global prevalence of diabetes. Estimates for the year 2000 and projections for 2030. Diabetes Care, 27 (5): 1047-1053. 